\title{
Venite A spasso con noi!
}

\section{Come On the road with us!}

\author{
Daniela Kappler \\ SUPSI, Dipartimento formazione e apprendimento \\ $\square$ daniela.kappler@supsi.ch
}

Riassunto / I percorsi didattici A spasso con noi rappresentano un'innovazione sul piano nazionale e internazionale per quanto concerne la declinazione concreta e multimodale del metodo Content and Language Integrated Learning (CLIL) a favore della lingua italiana e per allievi ${ }^{1}$ di scuola elementare. I materiali costituiscono i frutti di esperienze didattiche plurime con bambini e adulti e di intense collaborazioni intercantonali e sono dedicati a classi di scuola elementare del Canton Uri. Da agosto 2020 un connubio uni$\mathrm{co}$, virtuoso e coerente, di materiali cartacei e digitali ricchi di tasks che lasciano molto spazio alla personalizzazione e alla differenziazione è entrato nelle aule. II fil rouge che dischiude gli orientamenti del nuovo Piano di studio della Svizzera tedesca e del CLIL è, "naturalmente", il viaggio. Un viaggio alla scoperta del territorio e della lingua della Svizzera italiana. II presente articolo ripercorre le esperienze didattiche preparate e vissute all'interno del progetto e ne ritrae il valore formativo per le persone e le istituzioni coinvolte.

Parole chiave: italiano lingua straniera; progetto collaborativo-didattico; CLIL interculturale; multimodalità; scuola elementare.

Abstract / The teaching material A spasso con noi embodies a national and international innovation with regard to the concrete and multimodal application of the Content and Language Integrated Learning (CLIL) method for the Italian language and for primary school pupils. The material is the result of multiple teaching experiences with children and adults within the project as well as of intensive inter-cantonal cooperation and is ded-

1 Nel presente articolo, dove non specificato, si farà uso del genere maschile per scorrevolezza di lettura, intendendo in ogni caso sia il genere femminile che quello maschile.

(C) 2021 Daniela Kappler. Questo è un articolo Open Access, sottoposto a un processo di revisione tra pari a doppio cieco, pubblicato dal Centro competenze didattica dell'italiano lingua di scolarizzazione e dal Servizio risorse didattiche e scientifiche, eventi e comunicazione del DFA-SUPSI in collaborazione con l'Alta scuola pedagogica dei Grigioni. L'articolo è distribuito sotto i termini della Licenza Creative Commons Attribuzione - Non commerciale - Non opere derivate 4.0 Internazionale (CC BY-NC-ND 4.0). 
icated to primary school classes in the canton of Uri. Since August 2020, a unique, dynamic, virtuous and coherent combination of task-rich paper and digital materials that leave plenty of room for personalisation and differentiation has entered the classrooms. The red thread lead-ing to the achievement of principles and goals of the new Swiss-German curriculum and of the intercultural and interdisciplinary CLIL of $A$ spasso is, "of course", journey - a journey through the regions. A journey to discover the region and language(s) of the Italian-speaking Switzerland. This article traces the educational experiences prepared and lived within the project and portrays its educational value for the people and institutions involved.

Keywords: Italian as a foreign language; collaborative-didactic project; Intercultural CLIL; multimodality; elementary school.

\section{Introduzione e contesto}

Un'eccezionale combinazione di volontà a promuovere l'italiano nelle scuole elementari di un'area germanofona a nord dell'arco alpino svizzero da parte del Canton Uri, dell'Alta scuola pedagogica di Svitto, del dopo "Svizzera italiana" (d'ora in avanti DFA-SUPSI) ha dato vita a un progetto di ricerca e sviluppo denominato In viaggio con l'italiano. Avviato nel 2018 e sostenuto dagli enti menzionati e dall’Ufficio Federale della Cultura nel suo messaggio sulla cultura 2016-2020 per la promozione dell'italiano al di fuori della Svizzera italiana (Bundesamt für Kultur, 2016), il progetto prevede l'ideazione, creazione e sperimentazione di un materiale didattico nuovo e innovativo, composto da manuali cartacei e una piattaforma digitale con video, attività interattive, schede con esercizi e una guida per i docenti. Nel presente articolo tenteremo di riportare temi e momenti salienti del progetto e racconteremo le esperienze didattiche progettuali svolte sia a livello terziario che a livello di scuola elementare per giungere alla realizzazione dei prodotti menzionati.

Nel panorama multilingue e plurilingue svizzero, in cui ritroviamo tre grandi regioni linguistiche, germanofona, francofona e italofona, e delle aree circoscritte del romancio, il Canton Uri, cantone germanofono e uno dei primi tre cantoni della Confederazione Svizzera, si situa giusto oltre il passo del San Gottardo a nord del Ticino, il cantone italofono. 


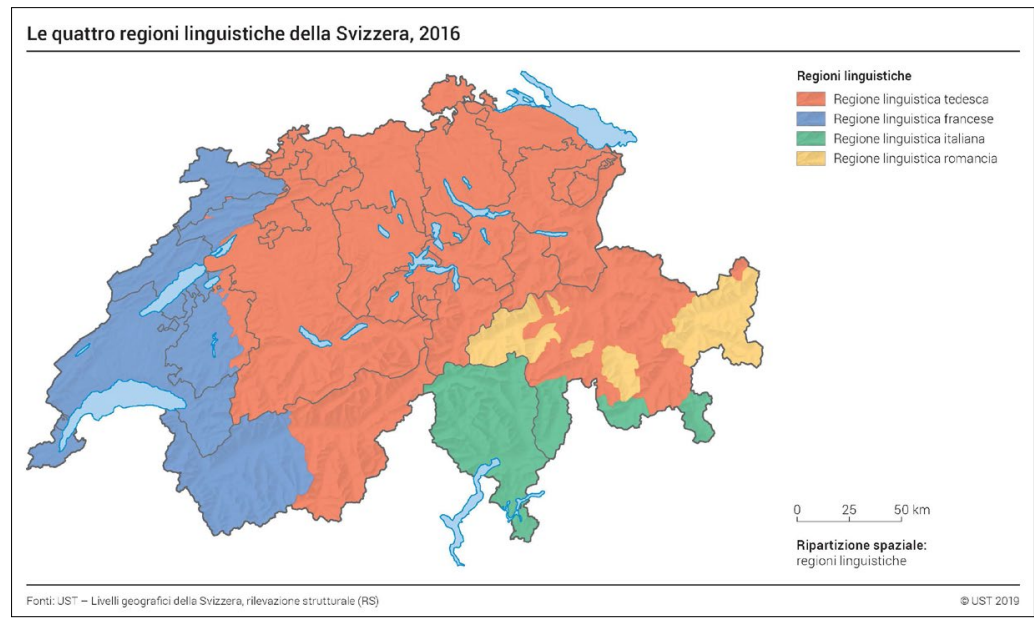

Figura 1. Mappatura delle lingue nazionali; tedesco, francese, italiano e romancio (fonte: https://www.plurilingua.admin.ch/plurilingua/it/home/themen/mehrsprachigkeit-der-schweiz.html).

Il Canton Uri vanta una lunga tradizione scolastica e politica di promozione dell'italiano come lingua straniera (1994) e, negli ultimi anni, una scelta consapevole di favorire l'italiano prima del francese lo distacca dal classico modello 3/5 dell'insegnamento linguistico in atto in Svizzera.

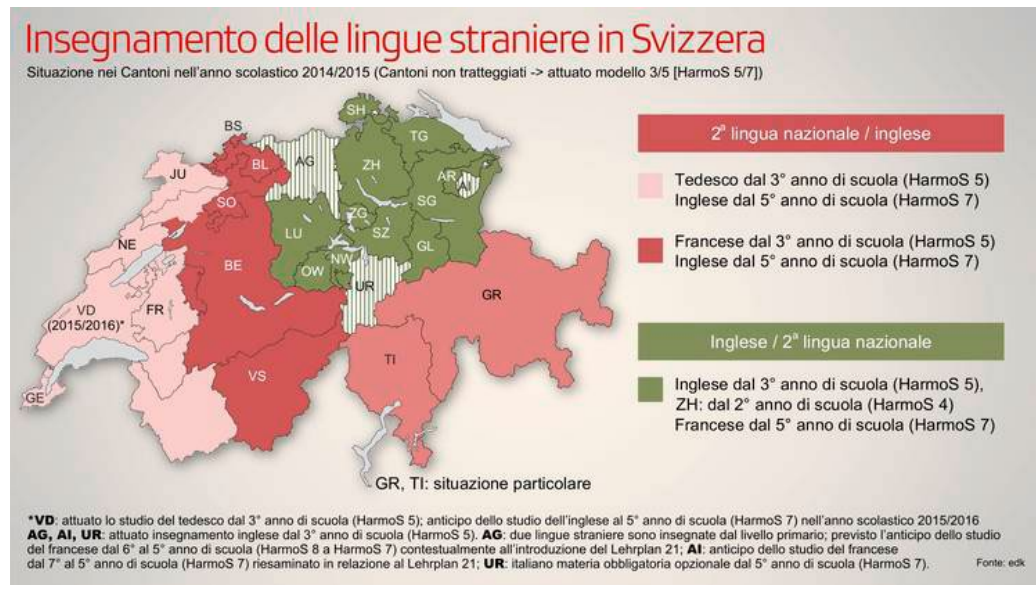

Figura 2. Mappatura delle lingue insegnate a scuola in Svizzera, dati del 2014-2015 (fonte: https://www.rsi.ch/news/svizzera/Zurigo-una-lingua-in-meno-6945479.html). 
In tale modello i cantoni germanofoni della Svizzera prevedono l'inglese o il francese come prima o seconda lingua straniera insegnata alla scuola elementare. In Svizzera, a livello di scuola elementare, solo in due cantoni si insegna l'italiano come lingua straniera: nel cantone trilingue dei Grigioni, sin dalla terza elementare, e nel Canton Uri, dalla quinta elementare, come materia opzionale. Secondo il concetto di insegnamento delle lingue straniere del Cantone, ciò significa che gli allievi, per la $5^{\mathrm{a}}$ e $6^{\mathrm{a}}$ elementare, devono scegliere se seguire un approfondimento di matematica e tedesco o imparare l'italiano, e che apprenderanno il francese solo dal $7^{\circ}$ anno, ( $1^{\circ}$ anno della scuola secondaria). Da anni l'italiano è scelto da circa $1 / 3$ degli allievi, i quali poi proseguono spesso con l'italiano anche alle medie. Tra tali allievi ci sono ragazzi di origine italofona e/o che hanno contatti ed esperienze di italiano grazie alla vicinanza con la Svizzera italiana (Canton Ticino) e con l'Italia. A volte l'italiano si insegna in pluriclassi ( $5^{\mathrm{a}}$ e $6^{\mathrm{a}}$ insieme).

\section{II progetto: dalle esperienze didattiche pregresse nella scuola urana alla concezione di A spasso con noi}

Dal 2012 docenti e allievi urani usavano uno dei pochi manuali creati per l'italiano lingua straniera alla scuola elementare e pubblicato in Italia per allievi di 8-10 anni. Tuttavia, una volta entrato in vigore il nuovo Piano di studio regionale (Lehrplan 21, d'ora in avanti LP21) nel 2017, ci si rese conto che il manuale non conteneva nessuna relazione con la Svizzera, poca aderenza con gli orientamenti del Piano di studio e con gli interessi degli allievi di quell'età. I docenti hanno spesso integrato delle situazioni di dialogo, dei brevi racconti o delle schede di lavoro alle varie unità presenti nel manuale, oppure si sono recati in gita in alcuni dei luoghi più conosciuti della Svizzera italiana come Bellinzona e Lugano.

Tener conto della situazione geolinguistica e scolastica del Canton Uri, delle esperienze didattiche e degli obiettivi del LP21 è vitale per l'ideazione e realizzazione di un progetto di sviluppo di materiale per l'insegnamento; per concepire obiettivi, temi, attività e anche il formato. Le due coordinatrici del progetto appartenenti rispettivamente all'Alta scuola pedagogica di Svitto (PHSZ), che forma i docenti del Canton Uri, e al DFA-SUPSI, hanno chiamato in causa docenti 
di italiano del Canton Uri, docenti del Canton Ticino, un team tecnico esperto in educazione ai media e tecnologie digitali nella formazione e un gruppo di studentesse delle due alte scuole pedagogiche.

\section{6 | Kulturen im Fokus}

\section{A | Kenntnisse}

1. Die Schülerinen und Schüler kennen einige Merkmale des italienischsprachigen Kulturraums sowie kulturelle Gemeinsamkeiten und Unterschiede.

\section{B | Haltungen}

1. Die Schülerinen und Schüler können über ihre Haltung gegenüber dem italienischsprachigen Kulturraum und gegenüber kultureller Vielfalt nachdenken.

\section{C | Handlungen}

1. Die Schülerinen und Schüler begegnen Menschen und Erzeugnissen des italienischsprachigen Kulturaums virtuell und real.

Quelle: Bildungs- und Kulturdirektion des Kantons Uri (Hrsg.) (2015). Lehrplan 21: Sprachen.

Figura 3. Estratto del Piano di studio regionale Lehrplan 21, Canton Uri.

(Piano di studio dei 21 cantoni germanofoni della Svizzera, fonte: https://ur.lehrplan.ch/).

Per venire incontro alla situazione di base e alle esigenze del LP21 concernenti lo sviluppo delle competenze linguistiche, interculturali e mediali, e del sapere, saper fare, e saper essere (come si vede nella Figura 3), e delle competenze trasversali quali lo sviluppo personale, la collaborazione, la comunicazione, il pensiero riflessivo e critico, il pensiero creativo e le strategie d'apprendimento, si è concepito un materiale che includesse diversi approcci pedagogico-didattici moderni (Hutterli, S., Stotz, D., \& Zappatore, D., 2008), come i seguenti: 
- l'approccio comunicativo-interazionale;

- la didattica plurilingue, didattica dell'intercomprensione, éveil aux langues;

- il Task-Based Learning (TBL);

- la progressione a spirale;

- l'educazione interculturale;

- l'educazione ai media.

Importanti anche le riflessioni e le declinazioni concernenti la differenziazione, la multimodalità e l'accessibilità.

Non solo, si è voluto dare un taglio accentuato all'apprendimento tra pari; all'apprendimento situato; a un metodo CLIL che fosse interdisciplinare (tra geografia, storia e scienze) e interculturale (parlando di luoghi ed esperienze proprie, avvicinandosi a e confrontandosi con l'alterità̀).

Il progetto pare davvero ambizioso e complesso nella sua declinazione pratica, soprattutto tenendo conto che la combinazione di approcci e metodi dovrà essere non solo coerente ma anche "semplificata"/accessibile ad allievi di 10 e 12 anni e ai loro docenti. D’altra parte, prendendo spunto principalmente dalle caratteristiche degli allievi (interessi e competenze/conoscenze pregresse) e dalla volontà di promuovere lingua e cultura della Svizzera italiana al di fuori dei suoi confini, ci è venuto in mente uno sfondo integratore "naturale": il viaggio. Un viaggio attraverso la Svizzera italiana, con gite oltre confine in Italia, raccontato e guidato da ragazzi della stessa età. Tale proposito non facilita la produzione del materiale didattico, ma lo rinsalda e lo legittima, in quanto l'apprendimento dovrebbe esser visto situato e come un'avventura, con task e uso della lingua contestualizzati ed esperienze memorabili che rimangono nel tempo (EDK, 2017), un coinvolgimento che porta dall'apprendimento all'acquisizione.

1 Tale approccio evolve da fondamenti ed esperienze CLIL; vedasi anche una panoramica storico-geografica: Maljers, A., Marsch, D., \& Wolff, D. (2007); Mehisto, P., Frigols, M.J., Marsh, D. (2008); Stotz, D., Bartholemy, C., \& Thonhauser, I. (2018). 


\section{Collaborazioni ed esperienze didattiche}

All'interno del progetto ci siamo avvalse prima di tutto della preziosa collaborazione di quattro docenti di scuola elementare e media ticinesi. In tal modo la Svizzera italiana funge non solo da oggetto di insegnamento, ma contribuisce direttamente alla creazione del materiale, tramite il coinvolgimento di attori scolastici reali che possono veicolare concreti aspetti linguistici e culturali. Importantissimi anche i quattro docenti urani, le formatrici e studenti/ esse di un corso in comunicazione visiva, i formatori e gli studenti di cinema. Alcune studentesse del DFA-SUPSI e della PHSZ hanno altresì collaborato al progetto con la ricerca di materiale fotografico, la presa di contatto con le associazioni del territorio, le traduzioni dei videoscript e lo sviluppo delle learning apps. La situazione pandemica iniziata in primavera 2020 ha purtroppo ostacolato e rallentato lo scambio e il lavoro didattico fra il gruppo di autori nonché la sperimentazione dei materiali e la produzione dei video per molti mesi.

\subsection{Esperienze con docenti, autrici e autori di $A$ spasso}

Nella prima fase del progetto, in qualità di coordinatrici del progetto, abbiamo cercato docenti ticinesi di scuola elementare e media interessati al progetto e reclutato coloro che riportassero non solo conoscenze del territorio e della lingua ma anche esperienze (e formazione) nell'insegnamento dell'italiano ad allievi alloglotti/stranieri. Insieme ai docenti di italiano del Canton Uri, i docenti ticinesi sono stati i primi interlocutori ai quali introdurre la ricca filosofia del progetto e, per questo, sono stati guidati nell'avvicinamento al metodo CLIL ancora poco diffuso alle elementari - soprattutto per quanto riguarda la concezione interculturale, annuale e interdisciplinare che lo caratterizza. Avremmo voluto avere dei veri e propri tandem tra i docenti in cui le idee e le conoscenze fossero un fruttuoso e immediato scambio di feedback e feedforward. A causa di differenti tradizioni didattiche tra nord e sud abbiamo dovuto optare per un'alternativa che comportasse più passaggi, tramite le due coordinatrici del progetto. Nella Svizzera tedesca i docenti delle scuole elementari hanno un manuale per ogni materia, mentre in Ticino solo per l'insegnamento della lingua straniera. Ciò implica un costante lavoro di ideazione e realizzazione individuale o in gruppo di docenti e di allievi di percorsi didattici per le loro classi e dunque 
una notevole differenza nell'esperienza di creare materiale didattico da singole unità o schede a interi percorsi e fascicoli. I docenti di Uri hanno svolto dunque un ruolo di consulenti a partire dal percorso generale fino agli schizzi di idee, ai percorsi messi su carta e ai video. Essi hanno inoltre sperimentato i materiali, con riscontri positivi e preziosi suggerimenti. I percorsi didattici vengono così adattati e migliorati, ad esempio anche con un interessante numero di file audio (testi, dialoghi) o con i temi per il classroom language, presentati all'inizio dei percorsi.

3.2 Esperienze con formatrici e studenti nel Bachelor in comunicazione visiva Per attuare gli obiettivi e i propositi menzionati in precedenza, abbiamo sentito la necessità di lavorare su un layout libero, ovvero su dei template non preimpostati da una casa editrice, in quanto di norma gli editori richiedono di sfruttare gli spazi e di riempirli il più possibile con degli esercizi. Con l'intenzione di volere un materiale didattico innovativo non solo dal punto di vista dell'approccio pedagogico-didattico ma anche da quello del formato, ci siamo rivolte al Dipartimento ambiente, costruzioni e design (DACD) della SUPSI. In accordo con le docenti del Corso di laurea in comunicazione visiva abbiamo lanciato il corso semestrale condividendo con le dieci studentesse e uno studente del secondo anno le personali esperienze di apprendimento delle lingue straniere a scuola (fino all'università) per poi passare all'analisi di una serie di manuali pubblicati per l'insegnamento di diverse lingue. Anche le docenti e le studentesse/lo studente hanno ritenuto che molti manuali tendono a essere troppo rigidi e densi di informazioni, sia scritte che visive (colori, foto, disegni, riquadri), un eccesso che distrae, che fa perdere l'efficacia ai fini dell'apprendimento. Hanno inoltre notato che vi sono pochissime occasioni autentiche per parlare, sia in lingua della classe che in lingua target, e che vi siano molte esercitazioni scritte, nonostante l'indicazione di un approccio comunicativo. Non da ultimo, il gruppo è rimasto sorpreso dal fatto che oggigiorno, per ogni anno scolastico, un volume didattico fosse molto corposo, composto da un libro per l'allievo, un libro per gli esercizi, un libro di lettura o civiltà, a cui spesso i docenti di lingue aggiungono un quaderno per il lessico e/o la grammatica e tante schede raccolte in un classificatore; 
un peso incredibile sulle spalle degli allievi, che già alle elementari seguono da una a due lingue straniere. L'obiettivo di voler sviluppare un materiale didattico volutamente leggero, che non si appiattisca sull'idea di libro o manuale da un lato e che integri in maniera estensiva e coerente diversi strumenti digitali, era dunque condiviso. Agli studenti è stato chiesto di elaborare una propria linea grafica, progettando e impaginando una parte del manuale, che lasciasse tanto spazio agli alunni e alla personalizzazione, con caratteristiche simili a uno scrapbook. Gli studenti dovevano inoltre considerare le necessità di adottare uno stile grafico uniforme e accessibile a una pluralità di allievi e di strumenti didattici, declinando la sua applicazione anche al sito web, ai video, agli esercizi e alla guida per i docenti. Una prima presentazione e condivisione delle idee progettuali era pianificata per la metà del semestre con il team di progetto e anche con una docente di sostegno pedagogico di Uri, mentre alla conclusione del semestre la versione grafica di A spasso con noi è stata selezionata durante una presentazione finale, in cui rientrava anche la valutazione complessiva. Tutto il gruppo ha potuto fare esperienza con un committente reale e la studentessa vincitrice ha ricevuto un primo mandato professionale per sviluppare la grafica di tutto il manuale.
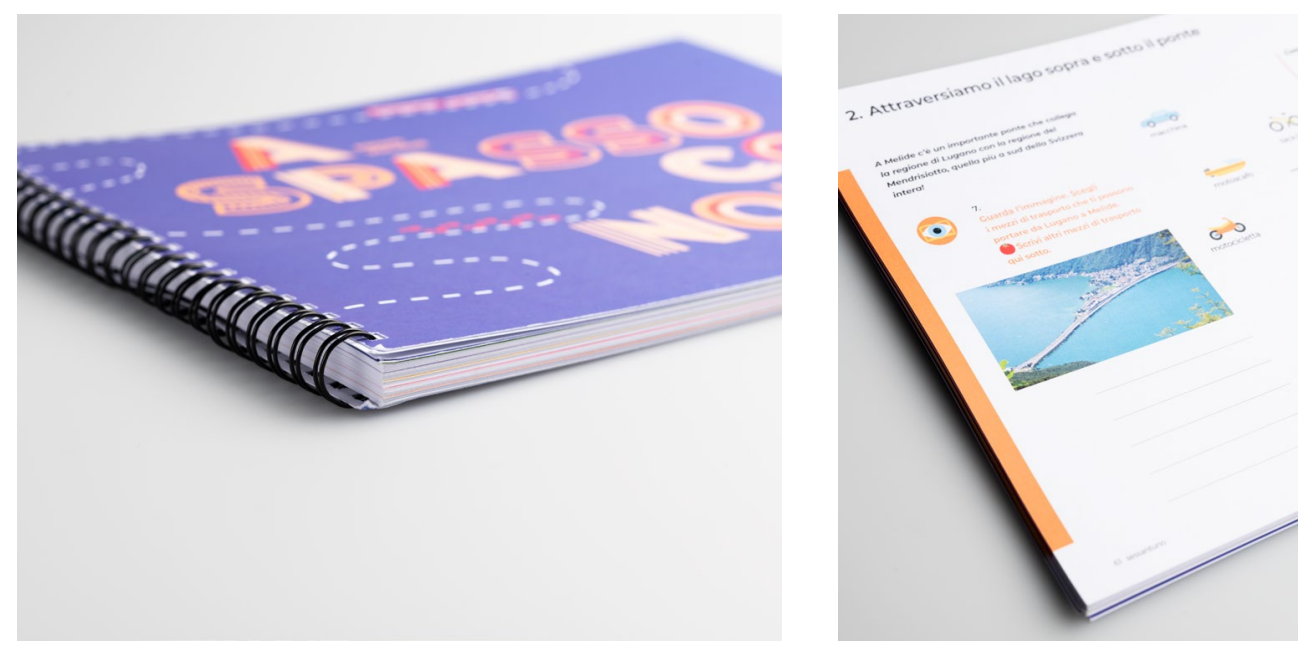

Figure 4 e 5. Manuale 1 per la $5^{\text {a }}$ elementare; leggero e rilegato a spirale e copertina in stile vintage; è come uno scrapbook che gli allievi possono personalizzare con le proprie idee, ricerche e creazioni (testi, immagini, disegni, dialoghi) (fonte: immagini scattate da studenti SUPSI/DACD). 
Una delle due formatrici del corso di laurea ha finalizzato il layout dei due manuali, preparando e inviando inoltre tutti gli elementi di grafica ai produttori dei video, al team tecnico per la creazione del sito e agli autori per creare il manuale 2 e tutte le schede. Per le coordinatrici, le autrici e gli autori è stata un'ottima occasione per approfondire importanti questioni di layout ai fini dell'apprendimento, completata con una formazione interna sull'accessibilità, svolta da un esperto collaboratore del governo.

\subsection{Esperienze con formatori e studenti del CISA}

Il CISALab del Conservatorio Internazionale di Scienze Audiovisive Film Academy di Locarno è stato l'interlocutore successivo. Avevamo l'idea di rappresentare virtualmente il viaggio nella Svizzera italiana in 20 brevi video, non come spot turistici, bensì come stimolo linguistico e culturale tramite l'accompagnamento di un gruppo di ragazzi che vivono nella Svizzera italiana e che hanno la stessa età. Questo percorso progettuale ha compreso la stesura degli script insieme ai docenti ticinesi, e poi l'organizzazione di un casting e di riprese, così come i lavori di pre- e post produzione con il CISALab. Durante questi compiti abbiamo imparato dei rudimenti del linguaggio cinematografico e a pensare agli elementi di scena necessari: dai diversi vestiti stagionali, agli accessori caratterizzanti dei ragazzi, alle musiche, agli intro ecc. Abbiamo optato per la disseminazione di volantini per il provino tramite le scuole, e per il primo manuale, abbiamo raccolto una ventina di ragazze e ragazzi tra i 10 e 13 anni interessati, mentre per il secondo manuale erano già una quarantina. Per ogni percorso didattico/manuale avevamo previsto un gruppo di cinque ragazzi, cercando alla fine un equilibrio di genere (cinque ragazze e cinque ragazzi). Le riprese per i 10 video del 2019 (manuale 1) e per i 10 video del 2021 (manuale 2) sono durate ogni volta un intero fine settimana, per i quali il CISALab ha montato uno schermo bianco nella grande sala di registrazione e posizionato luci, audio e cineprese. 

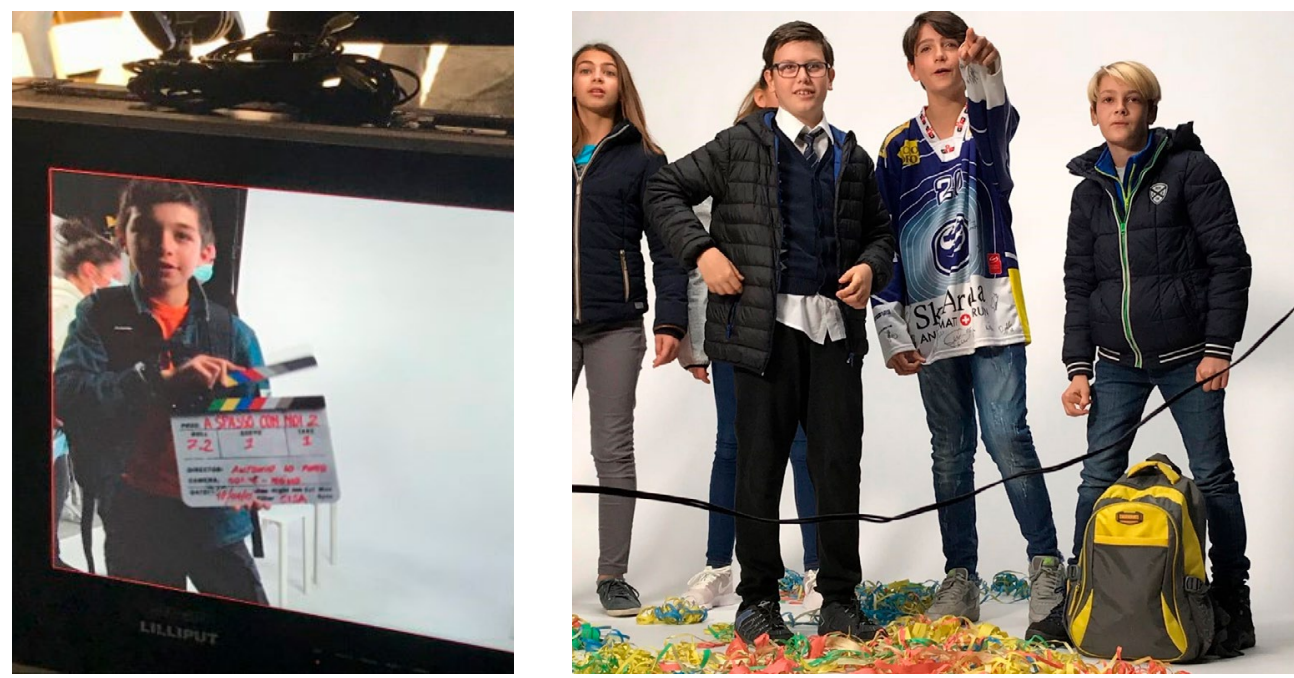

Figure 6 e 7. Momenti durante le riprese (fonte: immagini dell'autrice, Kappler, D. 2019 e 2021).

Lo schermo bianco permette in fase di post-produzione di inserire brevi frasi o pop up con immagini relative ai luoghi che si visitano e gli oggetti che si incontrano. Nel primo manuale i cinque ragazzi, che si sono conosciuti a un campo sportivo nella valle Leventina (che apre il percorso didattico del manuale 1) e che invitano gli allievi di Uri a venire a conoscere il Ticino e il Grigioni italiano, rappresentano regioni diverse da nord a sud della Svizzera italiana (il Bellinzonese, la Mesolcina, il Luganese eil Mendrisiotto). Il loro itinerario è suddiviso in cinque grandi unità didattiche, che corrispondono ad altrettante tappe del viaggio che attraversa tali regioni durante le quattro stagioni e arriva fino a Poschiavo (nel Grigioni italiano).

I dieci video prodotti hanno offerto un'intensa attività di educazione ai media e all'immagine anche ai nostri allievi ticinesi, nonché un'attenzione particolare all'espressione linguistica e gestuale, che non doveva essere né troppo veloce o enfatizzata, ma neppure troppo lenta da sembrare innaturale e noiosa.

Molto importante per una serie di motivi è stato integrare la vita reale dei ragazzi nella caratterizzazione dei personaggi, ad esempio nel primo percorso un ragazzo parla anche francese ed è un giovane campione ticinese di judo, e un altro ha origine italiane ed è arrivato da poco in Ticino. 
Nel secondo percorso, il plurilinguismo dei ragazzi è addirittura potenziato. Ripartendo dal Sud, più precisamente da Chiasso, comune confinante con l'Italia e porta di entrata di molte famiglie di origine straniera, si è trovato il luogo ideale per l'incontro con il secondo gruppo di ragazzi che parla il tedesco, l'inglese, lo spagnolo, il tigrino e il cinese, ma anche alcuni dialetti ticinesi, nonchè il Gurinertitsch, il dialetto svizzero tedesco di Bosco Gurin. È vero che nei percorsi l'italiano è valorizzato come lingua nazionale e regionale, e che i ragazzi ne offrono il modello linguistico, tuttavia la realtà scolastica è ben più ricca e variegata culturalmente. E quando la ragazza della Mesolcina dice di saper parlare anche il tedesco, ma poi fa dei piccoli errori, suggerisce che non dobbiamo avere paura di sbagliare.

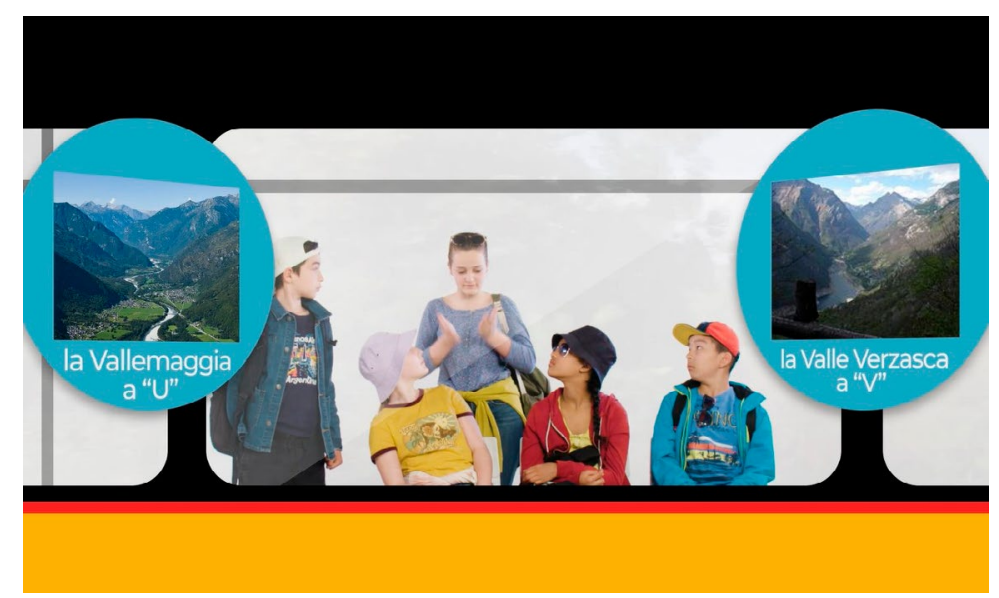

Figura 8. Estratto da un video della Tappa conclusiva (Tappa 10) (fonte: sito web www.aspasso.ch).

I video di A spasso con noi costituiscono sicuramente una delle componenti innovative nel panorama dei materiali didattici, tanto più nei materiali per la scuola elementare. Inoltre, gli studenti in regia e in tecnica di ripresa, del suono o dell'audio del CISA hanno potuto valorizzare tale collaborazione con il nostro progetto non solo come crediti formativi, ma anche come esperienza di lavoro con attori non professionisti, quindi con attori in erba, cioè ragazzi dell'età di 10-13 anni. Gli studenti hanno pubblicato un estratto del loro lavoro sul portale Vimeo, Youtube e Facebook (2019: https://vimeo.com/336321129, e 2021: https:// vimeo.com/628979728). 


\subsection{Esperienze con A spasso nelle scuole urane}

Prima e dopo l'introduzione del primo manuale a scuola quest'anno, abbiamo svolto dei pomeriggi di formazione continua insieme al dipartimento dell'educazione del Canton Uri. I docenti di italiano nel Canton Uri sono anche docenti di inglese o docenti di scuola elementare a metà tempo che dedicano l'altra metà ai corsi di italiano nelle classi $5^{\mathrm{a}}$ e $6^{\mathrm{a}}$ nella stessa scuola. Ci sono docenti che conoscono meglio l'Italia, in quanto vi hanno passato dei periodi di studio della lingua o di vacanza; l'approccio CLIL per conoscere la Svizzera italiana permette quindi anche a loro di esplorare e conoscere meglio dei territori italofoni nazionali. Attualmente la sfida didattica centrale è il concetto e la modalità di valutazione progressiva delle competenze linguistiche all'interno del metodo CLIL $^{2}$, a cui daremo tutti insieme un seguito nei prossimi pomeriggi di formazione. Si tratta di un procedere legittimo e piuttosto innovativo nel suo genere.

Grazie a visite in classe, a pomeriggi di formazione, a incontri formali e informali, a spontanei feedback scritti e a questionari possediamo una certa visione delle esperienze didattiche in classe. Alla prima lezione di italiano gli allievi urani scoprono una novità, un agile e maneggevole quaderno a spirale orientato orizzontalmente con il titolo che associano subito a un'attività piacevole, al divertimento (Spass in tedesco). Se in classe ci sono alunni con conoscenze dell'italiano, spiegano il senso ai compagni, indicando anche le impronte sulla via: andare a spasso significa uscire fuori a camminare ma anche divertimento: che spasso! Ecco il ricercato gioco di parole e uno dei messaggi del manuale già svelato. Non resta che interpretare con noi.

Ci sono docenti che introducono il percorso aprendo come prima cosa una grande cartina della Svizzera e chiedendo agli allievi di orientarsi tra i cantoni e le aree linguistiche. Le loro conoscenze pregresse e condivise trovano spazio fin dalle prime pagine del quaderno e anche lungo tutto il percorso. Poi, brevi testi in tedesco e simboli li introducono nello spirito del manuale; oltre alle classiche icone delle cinque abilità di base ci sono infatti tre icone che li invitano a guar-

2 Partendo ad esempio dalle utili indicazioni di Massler, U., \& Stotz, D. 2013. 
dare dei video, a cercare (sul web) e a creare da soli fai da te/selbermachen che implicano un apprendimento attivo.

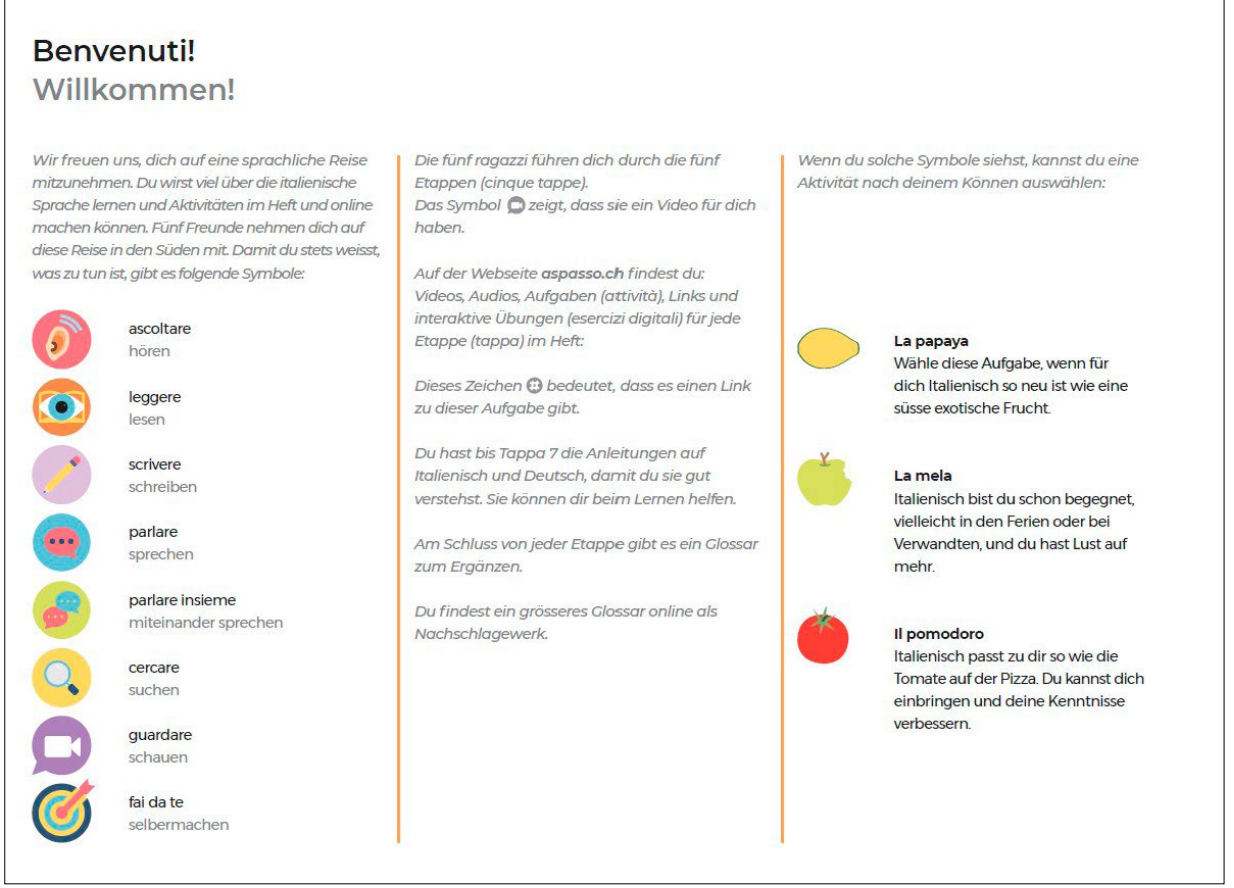

Figura 9. Estratto delle pagine introduttive del manuale/quaderno (fonte: Arnet, I., \& Kappler, D., 2020).

Docenti e allievi notano che la differenziazione è basata su conoscenze pregresse, non su (presunte) velocità di apprendimento o su (presunte) opinioni su cosa possa essere un esercizio difficile, medio o facile. In seguito, vedono come i temi e le attività spesso offrono formulazioni o task differenziati e così scelgono autonomamente, in gruppo o con il/la docente di classe come svolgerle. Si è notato che la differenziazione permette non solo alle pluriclassi di lavorare sullo stesso tema durante la fase di transizione dei manuali, ma anche agli allievi di origine italofona di avere il corso di italiano come un corso di lingua e cultura d'origine.

Quando gli allievi partono alla scoperta della Svizzera italiana, in tutte le dieci tappe vedono che il testo è sempre rivolto a loro, che siano brevi testi, titoli, sottotitoli, consegne; gli allievi sono sempre al centro; c’è un'azione 
da compiere, una frase da comunicare o una curiosità da scoprire. È chiaro per loro che sono i protagonisti fin dalla prima tappa. Inoltre, capiscono che entrano nella Svizzera italiana dal loro cantone, attraversando in diversi modi il San Gottardo, simbolo dell'incrocio tra Nord e Sud della Svizzera (e non solo). Entrando in Valle Leventina scoprono da un lato delle somiglianze con Uri, in quanto li unisce l'ambiente alpino, e ciò offre uno stimolo autentico per parlare del proprio ambiente, delle valli, dei villaggi, delle attività (sportive). Inoltre scoprono che i loro avi avevano per alcuni secoli il controllo del San Gottardo e della Valle Leventina e che sono rimaste delle tracce nella lingua e in alcuni edifici.

Nel complesso, capiscono facilmente che è presente un fil rouge e che specifiche illustrazioni rappresentano degli indizi per i temi che incontrano durante il percorso.

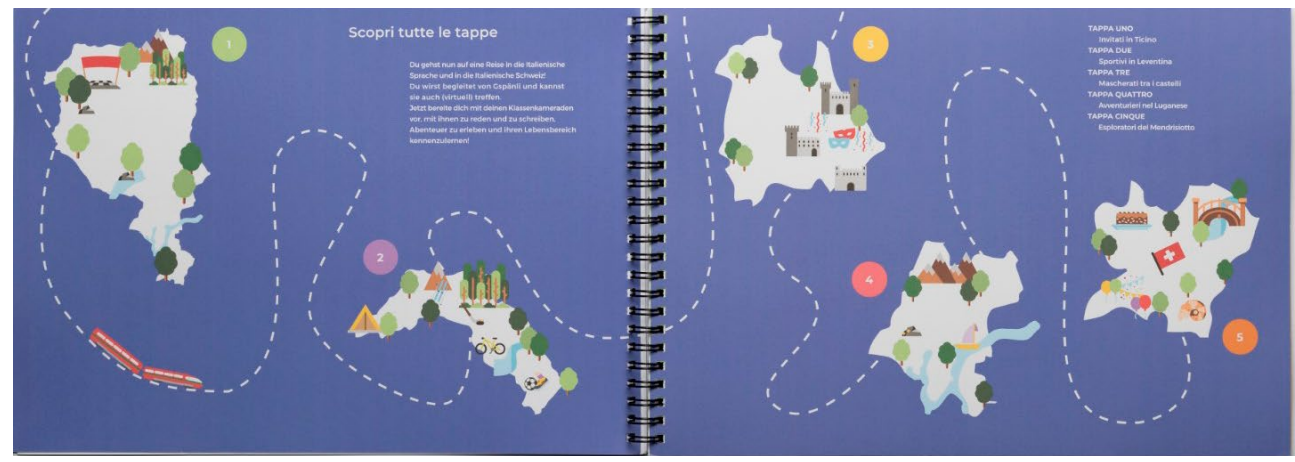

Figura 10. Estratto dell'indice tematico e geografico nel manuale 1 (fonte: Arnet, I., \& Kappler, D., 2020).

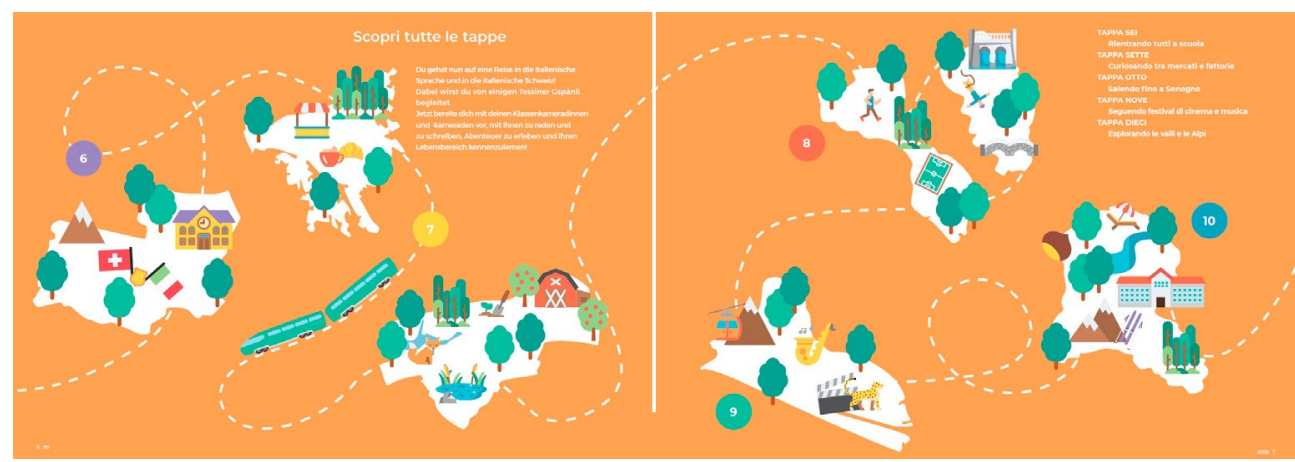

Figura 11. Estratto dell'indice tematico e geografico nel manuale 2 (fonte: Arnet, I., \& Kappler, D., 2021). 
Gli allievi che riprendono la scuola ad agosto partono dal punto più a Sud della Svizzera, più precisamente da Chiasso, comune confinante con l'Italia, e si vedono tematizzare non solo il multiculturalismo presente a scuola, ma anche il fatto che i loro coetanei in Ticino sono passati alla prima media, mentre loro vanno alla sesta elementare. Entrambi i temi incuriosiscono i ragazzi che riflettono su somiglianze e differenze socioculturali e scolastiche. I docenti, durante la sperimentazione del primo manuale, ci dicono di apprezzare gli stimoli di confronto, in particolare in attività che includano dei confronti tra le lingue e tra discipline (come nell'esempio di Figura 12). Essendo i docenti dei generalisti, riescono a vedere un argomento nella sua globalità, a fare connessioni interdisciplinari legate a un tema. Il CLIL interdisciplinare di A spasso si combina sia con le singole materie insegnate in lingua tedesca sia con gli approcci interdisciplinari dei docenti.

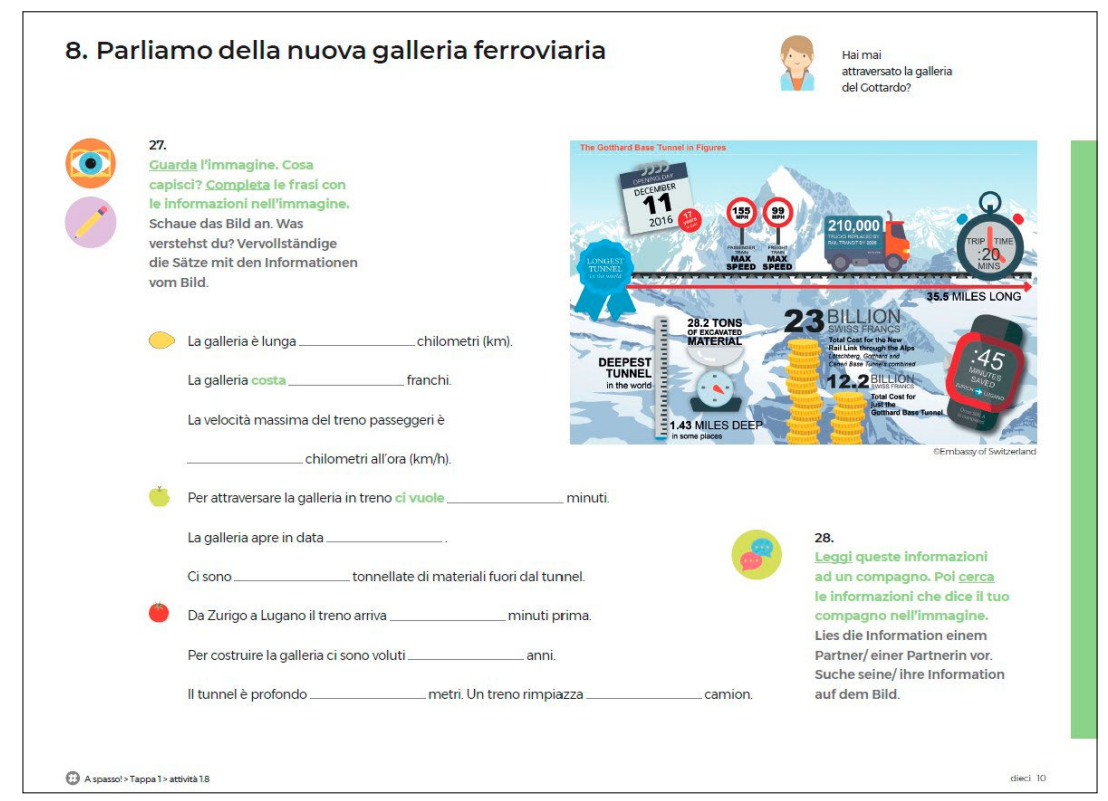

Figura 12. Estratto del manuale 1 in cui è presente il confronto tra unità di misura per rispondere ai quesiti differenziati (fonte: Arnet, I., \& Kappler, D., 2020). 
Il grande schermo e di tablet sono strumenti usati fin da subito in classe. Il sito www.aspasso.ch è a portata di mano. Gli allievi di Uri si accorgono immediatamente che ci sono due video per ogni tappa (episodio 1 nella prima parte ed episodio 2 in apertura della seconda parte) e soprattutto che sono in compagnia di ragazzi della loro età, che li invitano non solo nella Svizzera italiana ma anche a raccontarsi e a esprimere le loro emozioni ed esperienze.
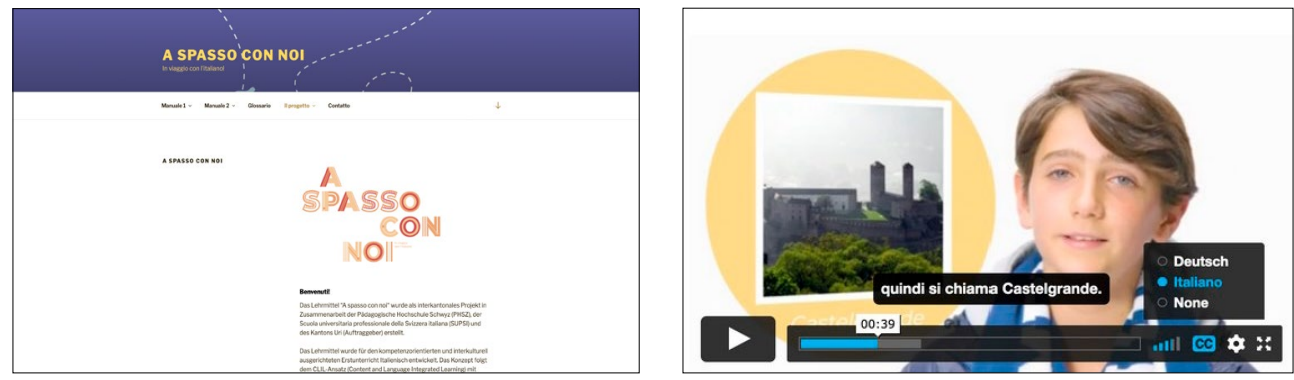

Figure 13 e 14. Il sito riprende la grafica del manuale, così come i video; qui si possono vedere i simboli, per vederli in classe come a casa, alla velocità normale o rallentata, con sottotitoli in italiano o tedesco (o senza) (fonte: sito web www.aspasso.ch).

\section{Conclusioni}

Il costante sostegno da parte del Canton Uri, così come la motivazione, la curiosità e l'impegno di docenti e allievi ad approcciarsi al mondo italofono nel suo contesto autentico e multiculturale costituiscono l'elemento fondamentale che ci ha spinto a realizzare questo progetto dal carattere innovativo e decisamente articolato. Con i nostri due grandi percorsi abbiamo concretizzato un nuovo e originale concetto integrato delle lingue, non solo quelle imparate a scuola prima o dopo l'italiano, ma anche le lingue d'origine dei ragazzi, che siano dialetti locali o lingue distanti (éveil aux langues). La didattica dell'intercomprensione è possibile anche con/tra lingue che non hanno per forza radici comuni, ma che con il contatto linguistico e diverse strategie diventano comprensibili. Nel nostro concetto di didattica plurilingue, l'intercomprensione è rivelata anche nelle consegne bilingui (fino alla tappa 4 nel manuale 1 e fino alla tappa 7 nel manuale 2), le quali per il metodo CLIL costituiscono non solo un utile strumento di scaffolding ma stimolano anche la mediazione linguistica. Inoltre, molte 
formulazioni delle consegne ritornano spesso - riproponendo quindi la ripresa a spirale non solo dei contenuti ma anche delle consegne - e sono accompagnate da icone. Per noi l'uso estensivo, la ricerca o la riproduzione di immagini sono a loro volta essenziali nell'insegnamento delle lingue e in particolare per l'applicazione del metodo CLIL. Pilotando i materiali, vediamo quanto tale quadro pedagogico-didattico sia fruttuoso.

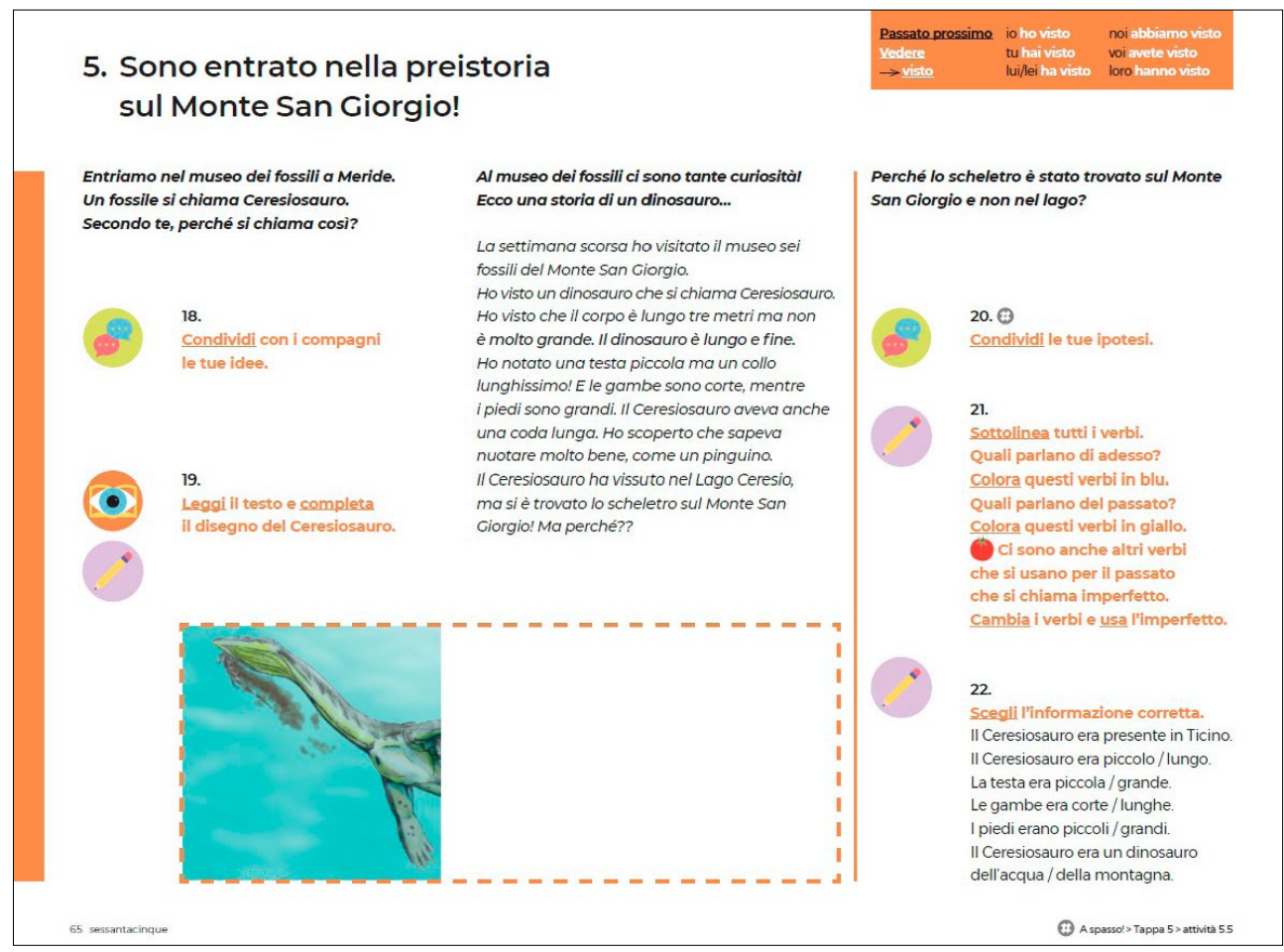

Figura 15. Esempio di CLIL contestualizzato con attenzione alla lingua (fonte: Arnet, I., \& Kappler, D., 2020).

Essendo nel nostro caso il CLIL inscritto nel contesto della Svizzera italiana, abbiamo preso contatto con molte associazioni ed enti del territorio, chiedendo loro una collaborazione e il permesso di usare immagini e/o video a scopo didattico (Ticino Turismo, Swissminiatur, le associazioni di hockey, l'associazione Rabadan di Bellinzona, la miniera d’oro di Sessa, il Locarno Film Festival, il Jazz Ascona, il Centro Sportivo nazionale di Tenero, l’associazione dei Ghiacciai 
di Poschiavo, teatri e musei). Il coinvolgimento diretto e spesso entusiasta di tali enti, così come di docenti e di famiglie, sportivi e attori, sindaci e direttori scolastici, ci ha permesso di offrire alle classi molte situazioni di contatto (dialoghi) e materiali autentici.

All'interno del progetto un principio è stato particolarmente impegnativo: trovare l'equilibrio nel CLIL tra task e attività prettamente linguistica, che sostengano entrambe le dimensioni che Cummins (2008) definisce BICS (Basic Interpersonal Communication Skills) e CALP (Cognitive Academic Language Proficiency, ad esempio Cummins, 2008), ovvero della lingua per comunicare nel quotidiano e della lingua più astratta e accademica. Ci era chiaro che i temi di geografia, scienze o storia contenuti nelle tappe del viaggio avrebbero assunto tutto il loro senso solo se integrati in un discorso coerente e contestualizzato in un ambiente, e non riprodotti come meteore sparse. Tuttavia, è solo cammin facendo, ideando e sperimentando che abbiamo trovato e troviamo una direzione efficace. Il ruolo dei ragazzi ticinesi riveste anche questo scopo; essi indicano la via per poter scoprire e conoscere luoghi, persone ed eventi, per poterne parlare, oralmente o per iscritto. E così anche i tempi verbali al passato, il passato prossimo in particolare, arrivano già “molto presto”, perché sono indispensabili per raccontare e raccontarsi. Ciò non significa che in sede di valutazione possiamo pretendere che gli allievi sappiano tutto ciò che è appena stato tematizzato. La progressione a spirale prende i suoi giusti tempi, include processi importanti di acculturazione e inculturazione, di riflessione esplicita sulla lingua, sulle strategie di apprendimento o sulle progressioni (feedback e feedforward) e porta al transfer linguistico e al plurilinguismo funzionale descritto e auspicato dal Quadro comune europeo per le lingue (Consiglio d'Europa, 2001) che guida i nostri piani di studio.

Dal nostro racconto dei momenti salienti del progetto si evince facilmente come, con le nostre idee, abbiamo coinvolto e incoraggiato tutto un mondo, anche diversi attori e settori che non si occupavano direttamente né di insegnamento dell'italiano come lingua straniera, né di manualistica o di progetti per la scuola dell'obbligo. E così, il progetto ha dato vita a un percorso di apprendimento, o meglio di acquisizione, a un ventaglio di protagonisti. 


\section{Bibliografia}

Arnet, I., \& Kappler, D. (2020). A spasso con noi. In viaggio con l'italiano. Volume 1. Kt. Uri: Druckerei Kuster.

Arnet, I., \& Kappler, D. (2021). A spasso con noi. In viaggio con l'italiano. Volume 2. Kt. Uri: Druckerei Kuster.

Bundesamt für Kultur (2016). Erhaltung und Förderung der rätoromanischen und der italienischen Sprache und Kultur. https://www.bak.admin.ch/bak/it/ home/sprachen-und-gesellschaft/lingue/uso-delle-lingue-ufficialidella-confederazione-e-promozione-del/salvaguardia-e-promozionedella-lingua-e-della-cultura-romancia-html

Consiglio d'Europa (2001). Quadro comune europeo per le lingue: apprendimento, insegnamento, valutazione. Milano: La Nuova Italia-Oxford.

Cummins, J. (2008). BICS and CALP: Empirical and Theoretical Status of the Distinction. In N. H. Hornberger (a cura di), Encyclopedia of Language and Education. Boston: Springer. https://doi.org/10.1007/978-0-387-30424-3 36

EDK (2017). Schweizerische Strategie-Austausch und Mobilität. https://www. edk.ch/de/themen/transversal/sprachen-und-austausch

Hutterli, S., Stotz, D., \& Zappatore, D. (2008). Do you parlez andere lingue? Fremdsprachen lernen in der Schule. Zürich: Verlag Pestalozzianum/HEP Verlag.

Kt. Uri. (2017). Lehrplan 21. https://ur.lehrplan.ch/

Maljers, A., Marsch, D., \& Wolff, D. (a cura di) (2007). Windows on CLIL. Content and Language Integrated Learning in the European Spotlight. The Hague, Alkmaar: European Platform for Dutch Education.

Massler, U., \& Stotz, D., (2013). CLIL_Unterricht in der Primarstufe. Ein theoriebasierter Leitfaden für die Entwicklung von Aufgaben für Unterricht und Beurteilung. Trier: Wissenschaftlicher Verlag Trier.

Mehisto, P., Frigols, M.J., \& Marsh, D. (2008). Uncovering CLIL. Oxford: Macmillan. 
Plurilingua (2021). https://www.plurilingua.admin.ch/plurilingua/it/home/themen/mehrsprachigkeit-der-schweiz.html

Stotz, D., Bartholemy, C., \& Thonhauser, I., (a cura di), (2018). Sprache und Sachfach integriert lernen: CLIL neu gedacht. Babylonia, 2018/2. Comano: Fondazione Lingue e Culture. 